\title{
Motor imagery classification in Brain computer interface (BCI) based on EEG signal by using machine learning technique
}

\author{
N. E. Md Isa, A. Amir, M. Z. Ilyas, M. S. Razalli
}

School of Computer and Communication Engineering, Universiti Malaysia Perlis (UniMAP), Malaysia

\begin{tabular}{l} 
Article Info \\
\hline Article history: \\
Received Oct 19, 2018 \\
Revised Nov 29, 2018 \\
Accepted Dec 14, 2018 \\
\hline
\end{tabular}

\section{Keywords:}

BCI

Classification

EEG

Machine learning

Motor imagery

\begin{abstract}
This paper focuses on classification of motor imagery in Brain Computer Interface (BCI) by using classifiers from machine learning technique. The BCI system consists of two main steps which are feature extraction and classification. The Fast Fourier Transform (FFT) features is extracted from the electroencephalography (EEG) signals to transform the signals into frequency domain. Due to the high dimensionality of data resulting from the feature extraction stage, the Linear Discriminant Analysis (LDA) is used to minimize the number of dimension by finding the feature subspace that optimizes class separability. Five classifiers: Support Vector Machine (SVM), K-Nearest Neighbors (KNN), Naïve Bayes, Decision Tree and Logistic Regression are used in the study. The performance was tested by using Dataset 1 from BCI Competition IV which consists of imaginary hand and foot movement EEG data. As a result, SVM, Logistic Regression and Naïve Bayes classifier achieved the highest accuracy with $89.09 \%$ in AUC measurement.
\end{abstract}

Copyright (c) 2019 Institute of Advanced Engineering and Science. All rights reserved.

\section{Corresponding Author:}

Nurul E'zzati Binti Md Isa,

School of Computer and Communication Engineering,

Universiti Malaysia Perlis,

02600 Arau, Perlis, Malaysia.

Email: nurulezzati@studentmail.unimap.edu.my

\section{INTRODUCTION}

Brain-computer Interface (BCI) is direct communication pathway between the brain and computer or other external equipment that can translate brain activities into commands [1-3] as shown in Figure 1. BCI has potential to become more popular in many application of various fields, for example, medical, entertainment and military [4]. There are many techniques to get the signals from the brain, such as electroencephalogram (EEG), Magnetic resonance imaging (MRI) and computer tomography (CT).

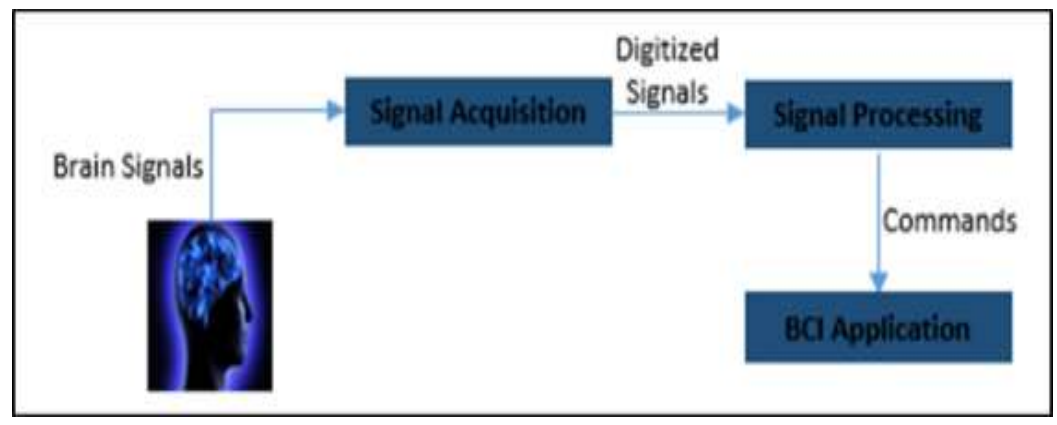

Figure 1. BCI system 
EEG is one of the most commonly used in BCI to records electrical activity and brain waves by placing the electrode on the scalp. EEG capable in capturing brain information processing quickly with high temporal resolution, but it has low spatial resolution and high noise level which make it challenging to extract useful information from EEG signals for BCI application [5].

Motor imagery is a popular paradigm in EEG-based BCI system [6]. The classification of the imaginary movement such as hand and foot movements is included in this paradigm [7]. Machine learning technique is commonly used in this classification process as it has the ability to model high-dimensional datasets [8]. Machine learning is the technique which can be briefly defined as enabling computers make successful predictions using past experiences [9].

In machine learning, there are various algorithms for classification process such as Support Vector Machine (SVM), K-Nearest Neighbors (KNN), Naïve Bayes, Decision Tree, and Logistic Regression. SVM is one of the algorithm that usually used for motor imagery classification in a BCI system [3, 6], [10-13]. In [14], the classification of motor imagery is done by comparing the signal using the KNN classifier. Besides that, KNN also used in automated seizure detection [15] to classify EEG signals either seizure or non-seizure. Naïve Bayes approach is used in [16, 17] to classify motor imagery and lower limb movement respectively. In [18], Decision Tree is used to classify the brain signals from imaginary tasks to open and close the hand for holding a ball. Logistic regression is used in [19] to classify the left and right motor imagery signals.

The successful deployment of a BCI system is depend on the effectiveness of signal processing to classify the desired signals. Therefore, the aim of this paper is to study the performance of various classification algorithms in machine learning technique which can be used to classify motor imaginary task. In this paper, we study five machine learning algorithm: SVM, KNN, Naïve Bayes, Decision Tree and Logistic Regression. The results helps in choosing better algorithm that makes a good classification performance in motor imagery classification. The rest of this paper is organized as follows: Section II gives the description of the dataset used. Section III contains the methodology of research. In section IV, the results is displayed with the discussion. Lastly, section $\mathrm{V}$ is conclusion.

\section{DATA DESCRIPTION}

The dataset used is Dataset 1 [20] from BCI Competition IV provided by B.Blankertz, C. Vidaurre and K.-R.Müller from Berlin (Germany). Motor imagery was performed by four healthy participants served as experimental subjects ( $\mathrm{a}, \mathrm{b}, \mathrm{f}$ and $\mathrm{g}$ ) [21]. Two mental tasks out of three tasks which are right hand movements, left hand movements or foot movements (side chosen by subject and can be both feet) is performed by the subjects in this experiment. The mental task performed by each subject is shown in Table 1 .

The experiment have two sessions which are calibration and evaluation session to record the training and test data respectively. Training data were provided with complete marker information which shown where the mental task is performed as it could be used for adapting the parameters of the methods or models while test data only consisted of the EEG signals, without any marker. Therefore, in this paper we use the data from calibration session only.

In the first two runs, all subjects was asked to perform a certain mental task based on arrows pointing left, right, or down that are presented as visual cues on a computer screen. The cues were displayed for a period of $4 \mathrm{~s}$ which interleaved with $2 \mathrm{~s}$ of blank screen and $2 \mathrm{~s}$ with a fixation cross shown in the center of the screen. Each subject have a total of 200 trials, as in each run 50 trials of each of the chosen two classes have been presented as shown in Table 2. A break of $15 \mathrm{~s}$ was given for relaxation after every 15 trials and there were longer breaks of $5-15 \mathrm{~min}$ between the runs.

Table 1. Mental task performed for each subject

\begin{tabular}{cc}
\hline Subjects & Movements \\
\hline a & Left hand, Right foot \\
b & Right hand, Left hand \\
f & Left hand, Right foot \\
g & Right hand, Left hand \\
\hline
\end{tabular}

Table 2. Total of trials for each of the chosen class

\begin{tabular}{cccc}
\hline Subjects & Movements & $1^{\text {st }}$ run & $2^{\text {nd }}$ run \\
\hline a,f & Left hand & 50 & 50 \\
& Right hand & 50 & 50 \\
b,g & Left hand & 50 & 50 \\
& Right hand & 50 & 50 \\
\hline
\end{tabular}

\section{RESEARCH METHOD}

\subsection{Feature extraction based on FFT-LDA}

The aim of feature extraction process is to extract the desired signals from the raw EEG signals and eliminates the unwanted signals such as background noise. The EEG signals is divided based on frequency bands which are gamma, beta, alpha, theta, and delta band. The frequency of motor imagery is usually lies in 
alpha or beta band. Thus, the EEG signal processing should be process in term of frequency. The raw EEG signals which is in time domain, need to convert to frequency domain for better extraction.

The Fast Fourier Transform (FFT) is applied in order to transforms the signal from the time domain to frequency domain. In FFT, the raw EEG signals is compared to sine waves consisting certain frequencies and the matching score is calculated. The result of matching score is depend on the similarity between the signals to the sine wave.

Linear Discriminant Analysis (LDA) is then applied to FFT features to increase the computational efficiency by reduce the number of dimension in a dataset. Therefore, the degree of over-fitting can be reduced and the class separability is optimized by finding the feature subspaces [22]. The features extracted from FFT-LDA are then feed into several classifiers for classification process. Figure 2 shows the block diagram of the feature extraction process for better understanding.

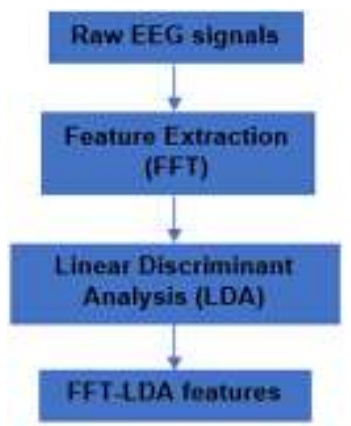

Figure 2. Block diagram of feature extraction process

\subsection{Classification}

Classification process is the technique to identify the class of the samples in the dataset. In this paper, the classifier is used to identify the type of movements such as left hand, right hand and right foot as shown in Figure 3. Five classifiers are used in this paper as follows:

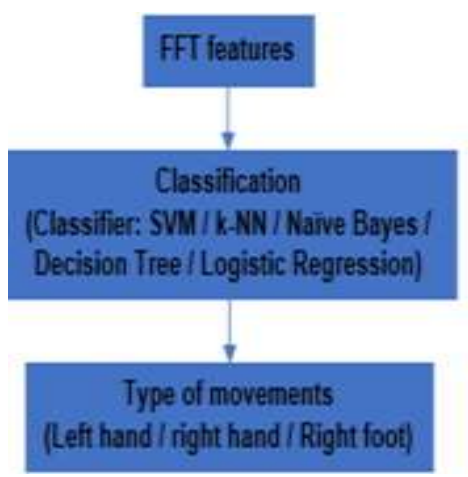

Figure 3. Block diagram of classification process

\subsubsection{Support Vector Machine (SVM)}

Support Vector Machine (SVM) is a classifier based on Statistical Learning Theory [23] which its algorithm is used to find decision boundary between the class samples, which correctly separates the samples into the classes. SVM can effectively prevent the defects of traditional classification methods, such as over learning, dimension disaster and local minima [3]. This classifier aims to maximize the distance between decision boundary and support vectors which is called as margin [22]. The samples is separates according to their class by SVM algorithm as this algorithm find the decision boundary between classes and maximize the margin.

\subsubsection{K-Nearest Neighbors $(\mathrm{KNN})$}

K-Nearest Neighbors (KNN) is a non-parametric learning algorithm [24] which capable to characterize EEG data as it is a suitable for noisy and large data and its result is depend on the value of $\mathrm{k}$ and 
distance metric used [23]. The new data point is classified by using the KNN algorithm with locates the $\mathrm{k}$ samples that are nearest to it based on the distance metric used and its class label is calculated depends on the class label of its k nearest neighbors.

The concept of KNN algorithm is illustrated in Figure 5. by using one of its distance metric. The symbol ' $X$ ' at point $(0.6,0.45)$ in the figure is shows the new data to be classified and the radius with the dot line is the result of applying KNN algorithm with $\mathrm{k}=9$ using Euclidean distance. In this case, there are two possible classes: circle class and triangle class. The KNN will classify the new data to the triangle class as the triangle class has the highest number of samples within the radius [7].

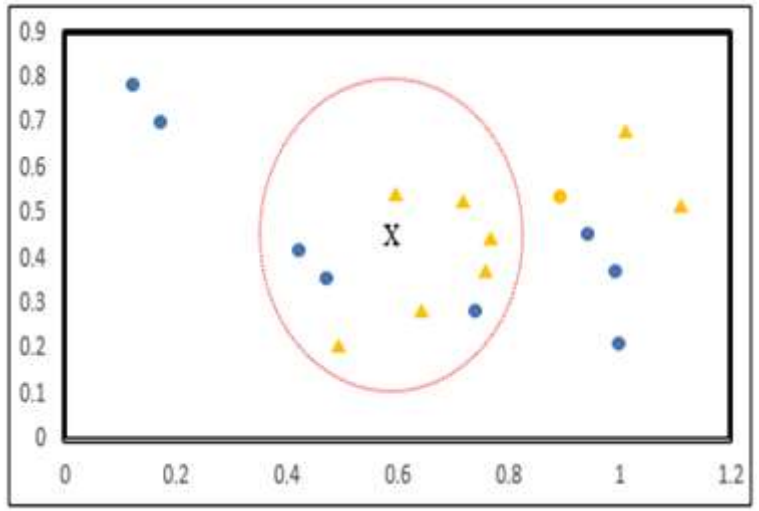

Figure 5. The KNN classification (euclidean distance with $\mathrm{k}=9$ ) [7]

\subsubsection{Naïve Bayes}

Bayes' theorem is used for this classifier [25] which calculates a set of probabilities by counting the frequency and combinations of values in a given dataset [26]. This algorithm is defined as;

$$
\mathrm{P}(C \mid X)=\frac{P(X \mid C) P(C)}{P(X)}
$$

where $\mathrm{P}(\mathrm{C} \mid \mathrm{X})$ denotes posterior probability which is the probability of class $\mathrm{C}$ given the data $\mathrm{X}, \mathrm{P}(\mathrm{C})$ denotes class prior probability which means the probability of class $C$ for the data $X$ being true, $P(X)$ denotes predictor prior probability which is the probability of the data regardless its class and $\mathrm{P}(\mathrm{X} \mid \mathrm{C})$ denotes likelihood which is the probability of the data $\mathrm{X}$ given that the class $\mathrm{C}$ was true.

\subsubsection{Decision tree}

Decision Tree classifier breaks down a dataset by splitting the dataset into two or more. This classifier works like a tree with root node, decision nodes and terminal nodes. The root node represents the entire dataset that will be split into two or more branch/sub-tree. The decision node is a node that will split into another sub-nodes and the terminal node which is also called as leaf node is represents a decision or classification that will not split further.

\subsubsection{Logistic regression}

Logistic Regression predicts the class of the data/sample by fitting data to the logistic function (inverse of logit function). The logit function takes input values in the range 0 and 1 and transforms them to values over the entire real number range, which we can use to express a linear relationship between feature values and the log-odds [22]:

$$
\operatorname{logit}(P(y=1 \mid x))=w_{0} x_{0}+w_{1} x_{1}+\cdots+w_{m} x_{m}=\sum_{i=0}^{m} w_{i} x_{i}=w^{T} x
$$

where $\mathrm{P}(\mathrm{y}=1 \mid \mathrm{x})$ is the conditional probability that are particular sample is belongs to class 1 given its feature $\mathrm{x}$. The inverse of logit function is called as logistic function, defined as;

$$
\emptyset(z)=\frac{1}{1+e^{-z}}
$$


Where $\mathrm{z}$ is the net input, the linear combination of weights and sample features can be calculated as;

$$
z=w^{T} x=w_{0}+w_{1} x_{1}+\cdots+w_{m} x_{m}
$$

\section{RESULTS AND ANALYSIS}

The classification of imaginary movements for each subject in this paper involves two classes: right hand vs left hand or left hand vs right foot. In this section, the classification result of imaginary movements by applying various classifiers in machine learning is tabulated and illustrated in tables and graphs respectively. The 10-fold cross validation technique is used to verify the results. The performance of classifiers is measure using accuracy and AUC (area under the curve). AUC used the concept of sensitivity and specificity which linked to true/false positives indices. Therefore it gives meaningful data compared to accuracy [27].

The result of the classifiers of all subjects is given in Table 3. It was observed that for classification between left hand and right foot using accuracy measurement, SVM give the highest result with $77.73 \%$ for the subject a and $71.18 \%$ for subject $\mathrm{f}$. In AUC measurement, SVM, Logistic Regression and Naïve Bayes give the highest result with $86.16 \%$ for the subject a and $80.02 \%$ for subject $\mathrm{f}$. For classification between right hand and left hand using accuracy measurement, Logistic Regression and Naïve Bayes give the highest accuracy, subject $\mathrm{b}$ with $66.34 \%$ and subject $\mathrm{g}$ with $79.23 \%$ respectively. In AUC measurement, SVM, Logistic Regression, Naïve Bayes give the highest accuracy with $72.20 \%$ for subject b and $89.09 \%$ for subject g. Figure 8 and Figure 9 shows the graph of comparison between accuracy and AUC measurement for left hand vs right foot and right hand vs left hand respectively.

Table 3. Result for subject a, $\mathrm{f}, \mathrm{b}$ and $\mathrm{g}$

\begin{tabular}{|c|c|c|c|c|c|c|c|c|}
\hline \multirow{4}{*}{ Classifier } & \multicolumn{4}{|c|}{ Left hand \& Right foot } & \multicolumn{4}{|c|}{ Left hand \& Right hand } \\
\hline & $\mathrm{a}$ & & $\mathrm{f}$ & & b & & $\mathrm{g}$ & \\
\hline & \multicolumn{8}{|c|}{ AccuracyAUCAccuracyAUCA } \\
\hline & $(\%)$ & $(\%)$ & $(\%)$ & $(\%)$ & $(\%)$ & $(\%)$ & $(\%)$ & $(\%)$ \\
\hline SVM & 77.73 & 86.16 & 71.18 & 80.02 & 66.01 & 72.20 & 78.89 & 89.09 \\
\hline $\mathrm{k}-\mathrm{NN}$ & 76.73 & 85.24 & 69.50 & 78.08 & 60.98 & 67.71 & 76.23 & 87.35 \\
\hline Logistic Regression & 77.40 & 86.16 & 71.01 & 80.02 & 66.34 & 72.20 & 78.73 & 89.09 \\
\hline Decision Tree & 77.40 & 84.60 & 67.82 & 75.94 & 64.01 & 69.08 & 76.21 & 87.48 \\
\hline Naïve Bayes & 77.40 & 86.16 & 71.01 & 80.02 & 65.68 & 72.20 & 79.23 & 89.09 \\
\hline
\end{tabular}

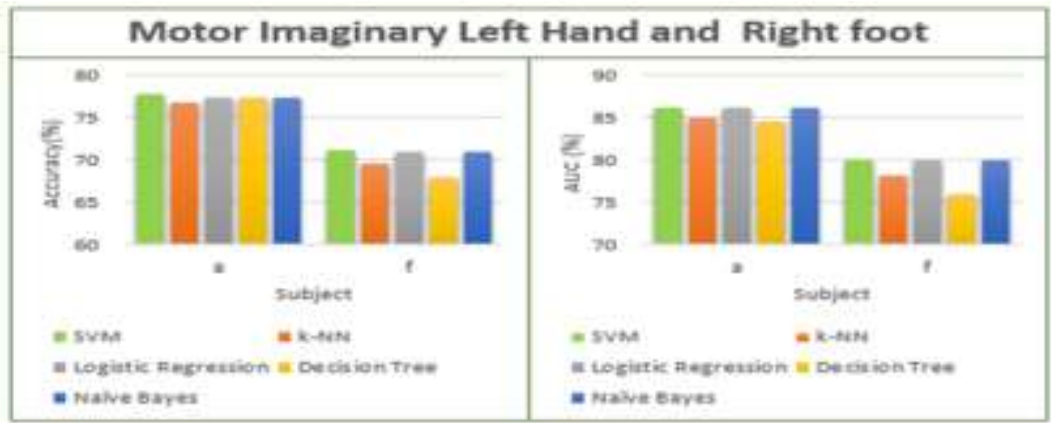

Figure 8. Accuracy and AUC measurement for motor imaginary left hand and right foot

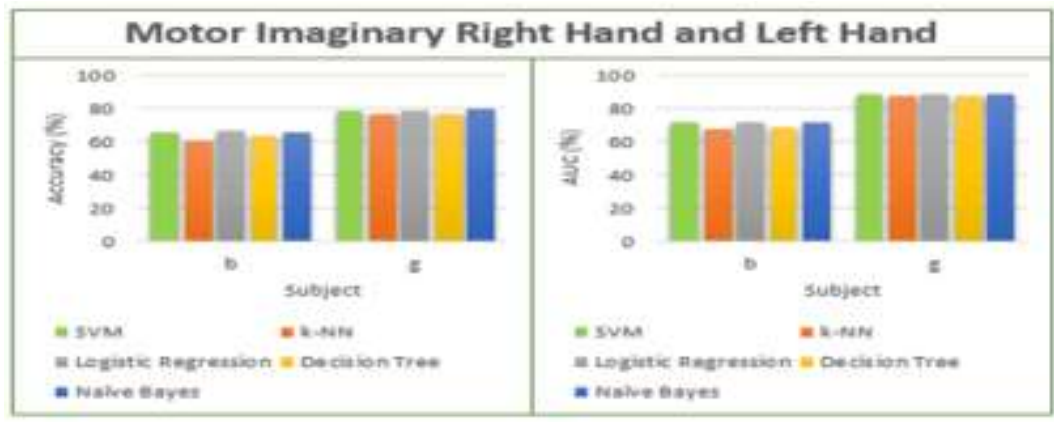

Figure 9. Accuracy and AUC measurement for motor imaginary right hand and left hand

Motor imagery classification in Brain computer interface (BCI) based on EEG... (N. E. Md Isa) 


\section{CONCLUSION}

This paper focuses on the classification of the EEG signal for imaginary movements to analyze the performance of classifiers from the machine learning technique. Feature extraction of the signals is done by using FFT-LDA technique. There is five classifier are used to classify the data which are SVM, k-NN, Logistic Regression, Decision Tree and Naïve Bayes. The AUC measurement gives a better result compared to accuracy measurement. Best results were obtained using SVM, Logistic Regression and Naïve Bayes classifier with $89.09 \%$ by using AUC measurement. In future work, we will investigate how to improve this algorithm in getting a better result than existing results for EEG classification.

\section{ACKNOWLEDGEMENTS}

The Dataset 1 of BCI Competition IV is provided by Berlin BCI group (Germany). In addition, the author would like to acknowledge the support from the Research Acculturation Grant Scheme (RAGS) (Grant No: 9018-0080) and Fundamental Research Grant Scheme (FRGS) under a grant number of FRGS/1/2015/ICT02/UNIMAP/01/1 from the Ministry of Higher Education Malaysia.

\section{REFERENCES}

[1] B. Yang, H. Li, Q. Wang, and Y. Zhang, "Subject-based feature extraction by using fisher WPD-CSP in braincomputer interfaces," Comput. Methods Programs Biomed. vol. 129, pp. 21-28, 2016.

[2] Z. Qiu, J. Jin, H. K. Lam, Y. Zhang, X. Wang, and A. Cichocki, "Improved SFFS method for channel selection in motor imagery based BCI," Neurocomputing, 2015.

[3] M. Ming, L. Shaona, M. Haitao, M. Yuliang, and G. Yunyuan, "Feature extraction method of motor imagery EEG based on DTCWT sample entropy," in 34th Chinese Control Conference (CCC), 2015, pp. 3964-3968.

[4] X. Tang, N. Zhang, J. Zhou, and Q. Liu, "Hidden-layer visible deep stacking network optimized by PSO for motor imagery EEG recognition,” Neurocomputing, vol. 234, no. April 2016, pp. 1-10, 2016.

[5] B. Yang, L. He, Q. Wang, C. Song, and Y. Zhang, "A preprocessing method of EEG based on EMD-ICA in BCI," Commun. Comput. Inf. Sci., vol. 461, pp. 1-12, 2014.

[6] J. Hong, X. Qin, J. Bai, P. Zhang and Y. Cheng, "A combined feature extraction method for left-right hand motor imagery in BCI," 2015 IEEE International Conference on Mechatronics and Automation (ICMA), Beijing, 2015, pp. 2621-2625.

[7] N. E. Md Isa, A. Amir, M. Z. Ilyas, and M. S. Razalli, "The Performance Analysis of K-Nearest Neighbors (K-NN) Algorithm for Motor Imagery Classification Based on EEG Signal," in MATEC Web of Conferences, 2017, vol. 140, p. 1024.

[8] A. Abraham et al., "Machine Learning for Neuroimaging with Scikit-Learn," Frontiers in neuroinformatics. vol. 8, no. February, pp. 1-10, 2014.

[9] A. Smola and S. V. N. Vishwanathan, "Introduction to machine learning," Methods Mol. Biol., vol. 1107, pp. 105-128, 2014.

[10] J. Feng et al., "Towards correlation-based time window selection method for motor imagery BCIs," Neural Networks, vol. 102, pp. 87-95, 2018

[11] S. Kumar, A. Sharma, and T. Tsunoda, "An improved discriminative filter bank selection approach for motor imagery EEG signal classification using mutual information,” BMC Bioinformatics, vol. 18, 2017.

[12] Z. Qiu, J. Jin, H. K. Lam, Y. Zhang, X. Wang, and A. Cichocki, "Improved SFFS method for channel selection in motor imagery based BCI," Neurocomputing, vol. 207, pp. 519-527, 2016.

[13] M. E. A. Abdel-Hadi, R. A. El-Khoribi, M. I. Shoman, and M. M. Refaey, "Classification of motor imagery tasks with LS-SVM in EEG-based self-paced BCI," 5th Int. Conf. Digit. Inf. Process. Commun. (CDIPC), pp. 244-249, 2015.

[14] J. Kevric and A. Subasi, "Comparison of signal decomposition methods in classification of EEG signals for motorimagery BCI system,” Biomed. Signal Process. Control, vol. 31, pp. 398-406, 2017.

[15] J. Birjandtalab, M. Baran Pouyan, D. Cogan, M. Nourani, and J. Harvey, "Automated seizure detection using limited-channel EEG and non-linear dimension reduction,” Comput. Biol. Med., vol. 82, pp. 49-58, 2017.

[16] Siuly, H. Wang, and Y. Zhang, "Detection of motor imagery EEG signals employing Naïve Bayes based learning process," Meas. J. Int. Meas. Confed., vol. 86, pp. 148-158, 2016.

[17] A. Rakshit, A. Khasnobish, and D. N. Tibarewala, "A Nä̈ve Bayesian Approach to Lower Limb Classification from EEG Signals," in 2016 2nd International Conference on Control, Instrumentation, Energy and Communication, CIEC 2016, 2016, pp. 140-144.

[18] D. F. Adamatti, J. Silveira, and F. Carvalho, "Analyzing Brain Signals Using Decision Trees: An Approach Based On Neuroscience," Rev. Eletrônica Argentina-Brasil Tecnol. Da Informação e da Comun., vol. 1, no. 5, 2016.

[19] J. Camacho and V. Manian, "Real-Time Single Channel EEG Motor Imagery based Brain Computer Interface," in World Automation Congress (WAC), 2016, pp. 1-6.

[20] B. Blankertz, G. Dornhege, M. Krauledat, K. R. Müller, and G. Curio, "The non-invasive Berlin Brain-Computer Interface: Fast acquisition of effective performance in untrained subjects," Neuroimage, vol. 37, no. 2, pp. 539-550, 2007. 
[21] M. Tangermann et al., "Review of the BCI competition IV," Frontiers in Neuroscience, no. JULY. 2012.

[22] S. Raschka, Python Machine Learning. 2016.

[23] R. Bose, A. Khasnobish, S. Bhaduri, and D. N. Tibarewala, "Performance Analysis of Left and Right Lower Limb Movement Classification from EEG," in 2016 3rd International Conference on Signal Processing and Integrated Networks (SPIN), 2016, pp. 174-179.

[24] S. K. Bashar, A. R. Hassan, and M. I. H. Bhuiyan, "Identification of motor imagery movements from EEG signals using Dual Tree Complex Wavelet Transform," 2015 Int. Conf. Adv. Comput. Commun. Informatics, pp. 290-296, 2015.

[25] M. H. Zaky, M. E. Khedr, and A. A. Nasser, "Effect of extensive training load on the classification accuracy for a three class motor imagery based brain-computer interface," in 2016 3rd International Conference on Advances in Computational Tools for Engineering Applications, ACTEA 2016, 2016, pp. 211-215.

[26] T. R. Patil and S. S. Sherekar, "Performance Analysis of Naive Bayes and J48 Classification Algorithm for Data Classification," Int. J. Comput. Sci. Appl. ISSN 0974-1011, vol. 6, no. 2, pp. 256-261, 2013.

[27] W. A. W. Azlan, S. H. Liew, Y. H. Choo, H. Zakaria, and Y. F. Low, "Wavelet feature extraction and J48 decision tree classification of auditory late response (ALR) elicited by transcranial magnetic stimulation," ARPN J. Eng. Appl. Sci., vol. 11, no. 10, pp. 6319-6323, 2016.

\section{BIOGRAPHIES OF AUTHORS}
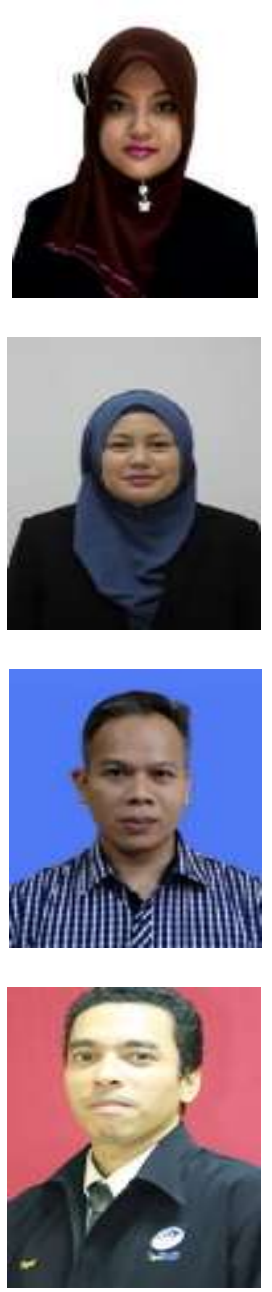

Nurul E'zzati Md Isa receives Malaysia Matriculation programme certificate from Perak Matriculation College in 2012, Bachelor of Engineering (Honors) in Computer Network Engineering from Universiti Malaysia Perlis (UniMAP) in 2016. She is currently pursuing the M.Sc. in Computer Engineering at UniMAP. Her current research interests include braincomputer Interface (BCI), EEG signal processing and machine learning technique.

Amiza Amir is a senior lecturer in School of Computer and Communication Engineering at Universiti Malaysia Perlis. She received her Ph.D. in Information Technology, on distributed artificial intelligence, from Monash University, Australia in 2015. Her current research interests include machine learning, distributed system, meta heuristic optimization and software-defined network (SDN). She teaches courses in data analytics and artificial intelligence.

Mohd Zaizu Ilyas received an associate degree in electrical engineering from Tsuyama National College of Technology Japan in 1998, B.Sc degree in electronics and information engineering from Tokyo University of Agriculture and Technology (TUAT) Japan in 2000 and M.Sc. in electric, electronics and system engineering from Universiti Kebangsaan Malaysia (UKM). He is currently pursuing the Ph.D. in Universiti Malaysia Perlis (UniMAP). His research interests include brain-computer Interface (BCI), EEG signal processing and speech processing.

Mohammad Shahrazel Razalli receives Diploma in Electrical and Electronic Engineering from Mara Institute of Technology Malaysia in 1993, Bachelor of Engineering (Honours) in Electronic Engineering from University of Leeds, United Kingdom in 1995, Master of Science in Communication and Network Engineering from Universiti Putra Malaysia in 2006 and Ph.D Communication and Network Engineering from Universiti Putra Malaysia in 2010. Currently, he is a senior lecturer at School Computer and Communication Engineering Universiti Malaysia Perlis. His research interests are amplifier, radio frequency and microwave circuits. 